\title{
An Enhanced Implementation of Brain Tumor Detection Using Segmentation Based on Soft Computing
}

\author{
T.Logeswari and M.Karnan
}

\begin{abstract}
Image Segmentation is an important and challenging factor in the medical image segmentation. This paper describes segmentation method consisting of two phases. In the first phase, the MRI brain image is acquired from patients database, In that film artifact and noise are removed. After that Hierarchical Self Organizing Map (HSOM) is applied for image segmentation. The HSOM is the extension of the conventional self organizing map used to classify the image row by row. In this lowest level of weight vector, a higher value of tumor pixels, computation speed is achieved by the HSOM with vector quantization.
\end{abstract}

Index Terms - Image analysis, segmentation, HSOM, tumor detection.

\section{INTRODUCTION}

Brain tumor is one of the major causes for the increase in mortality among children and adults. A tumor is a mass of tissue that grows out of control of the normal forces that regulates growth [22].. In India, totally 80,271 people are affected by various types of tumor (2007 estimates).. "Artificial Neural Networks (ANNs) are mathematical analogues of biological neural systems, in the sense that they are made up of a parallel interconnected system of nodes, called neurons. The parallel action is a difference between von Neumann computers and ANNs. Combining ANN architectures with different learning schemes, results in a variety of ANN systems. The proper ANN is obtained by taking into consideration the requirements of the specific application, as each ANN topology does not yield satisfactory results in all practical cases. The evolution of digital computers as well as the development of modern theories for learning and information processing led to the emergence of Computational Intelligence (CI) engineering. ANNs, Genetic Algorithms (GAs) and Fuzzy Logic are CI non-symbolic learning approaches for solving problems Mantzaris et al, 2008. The huge mass of applications, which ANNs have been used with satisfactory results, has supported their rapid growth. Fields that ANNs were used are image processing Gendy et al, 2001[13], environmental problems Bandyopadhyay and Chattopadhyay[10] Chattopadhyay and Chattopadhyay, [11], Climate study Chattopadhyay, [12], financial analysis Papadourakis et al,

T.Logeswari is a Research Scholar, with the Dept of computer Science, Mother Teresa Women's University,Kodaikanal, India(email: Saralogu4uin@gmail.com).

M.KARNAN is with the department of Computer Science and Engineering,Tamilnadu College of Engineering,Coimbatore, India(email: drmkarnan@gmail.com).
[9]. In this paper, a new unsupervised learning Optimization algorithms such as SOM are implemented to extract the suspicious region in the Segmentation of MRI Brain tumor. The textural features can be extracted from the suspicious region to classify them into benign or malign.

\section{IMAGE ACQUISITION}

The development of intra-operative imaging systems has contributed to improving the course of intracranial neurosurgical procedures. Among these systems, the $0.5 \mathrm{~T}$ intra-operative magnetic resonance scanner of the Kovai Medical Center and Hospital (KMCH, Signa SP, GE Medical Systems) offers the possibility to acquire $256 * 256 * 58(0.86 \mathrm{~mm}, \quad 0.86 \mathrm{~mm}, \quad 2.5 \mathrm{~mm}) \mathrm{T} 1$ weighted images with the fast spin echo protocol $(\mathrm{TR}=400, \mathrm{TE}=16 \mathrm{~ms}$, $\mathrm{FOV}=220 * 220 \mathrm{~mm})$ in 3 minutes and 40 seconds. The quality of every $256^{*} 256$ slice acquired intra-operatively is fairly similar to images acquired with a $1.5 \mathrm{~T}$ conventional scanner, but the major drawback of the intra-operative image is that the slice remains thick $(2.5 \mathrm{~mm})$.

\section{PREPROCESSING}

The MRI image consist of film artifact or labels on the MRI such as patient name, age and marks. Film artifact

That are removed using tracking algorithm. Here, starting from the first row and the first column, the intensity value, greater than that of the threshold value is removed from MRI. The high intensity value of film artifact are removed from MRI brain image. During removal of film artifacts, the image consist of salt and pepper noise.

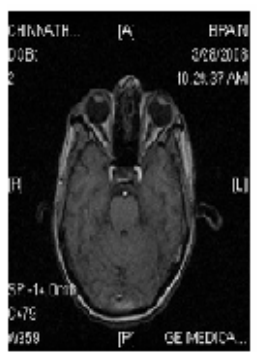

Before

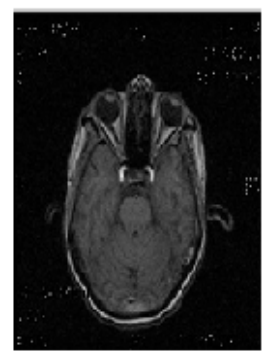

After
Preprocessing Preprocessing

Figure: Removal of film artifacts

The image is given to enhancement stage for the removing high intensity component and the above noise. This part is used to enhance the smoothness towards piecewise- homogeneous region and reduce the edgeblurring effects. This proposed system describe the 
information of enhancement using weighted median filter for removing high frequency component .

\section{Som \& Hsom Image Segementation}

A self-organizing map (SOM) or self-organizing feature map (SOFM) is a type of artificial neural network for unsupervised learning. SOMs operate in two modes: training and mapping, Training is a competitive process, also called vector quantization. Mapping automatically classifies a new input vector. Segmentation is an important process to extract information from complex medical images. Segmentation has wide application in medical field [2,14,21,22].

The main objective of the image segmentation is to partition an image into mutually exclusive and exhausted regions such that each region of interest is spatially contiguous and the pixels within the region are homogeneous with respect to a predefined criterion. Widely used homogeneity criteria include values of intensity, texture, color, range, surface normal and surface curvatures. During the past many researchers in the field of medical imaging and soft computing have made significant survey in the field of image segmentation $[1,7,17,25]$. Several authors suggested various algorithms for segmentation [15, 3, 23,27]. Suchendra et al, Multiscale image segmentation using a hierarchical self-organizing map[30 ] .

Murugavalli1 and Rajamani, A high speed parallel fuzzy c-mean algorithm for brain tumor segmentation [28]. Murugavalli1 and Rajamani, An Improved Implementation of Brain Tumor Detection Using Segmentation Based on Neuro Fuzzy Technique [29], Dana et al. designed a method on 3D Variational Segmentation for process due to the high diversity in appearance of tumor tissue from various patients [5].Image segmentation techniques can be classified as based on edge detection, region or surface growing, threshold level, classifier such as Hierarchical Self Organizing Map (HSOM), and feature vector clustering or vector quantization. The Trained Vector quantization has proved to be a very effective model for image segmentation process [4]. Vector quantization is a process of portioning ndimensional vector space into $M$ regions so as to optimize a criterion function when all the points in each region are approximated by the representation vector $\mathrm{Xi}$ associated with that region.

There are two processes involved in the vector quantization: one is the training process which determines the set of codebook vector according to the probability of the input data, the other is the encoding process which assigns input vectors to the code book vectors. Vector quantization process has been implemented in terms of the competitive learning neural network (CLNN)[6,26]. Self Organizing Map (SOM)[21] is a member of the CLNNs and this can be the best choice when implementing vector quantization using neural network[19]. The importance of SOM for vector quantization is primarily due to the similarity between the competitive learning process employed in the SOM and the vector quantization procedure. The main shortcoming of the SOM is that the number of neural units in the competitive layer needs to be approximately equal to the number of regions desired in the segmented image. The HSOM directly address the aforesaid shortcomings of the SOM. HSOM is the combination of self organization and graphic mapping technique.. The abstraction tree bears some resemblance to the major familiar quad tree data structure $[20,24]$ used in the several image processing and image analysis algorithms. In this paper, we propose a hybrid technique combining the advantages of HSOM was implemented for the MRI image segmentation

Overview Of Proposed Work

This paper describes the method of MRI brain image segmentation using Hierarchical self organizing map(HSOM) The below figure shows the flow of work in HSOM. In image acquisition process MR brain image is loaded into MATLAB 7.0.in the form of matrix

Next initialize the variables sigma, weight vector and winning neuron .In that Calculate the neighborhood function, weight vector and winning neuron .Here neuron is the input and winning neuron is the output. In that we find the adaptive threshold if the (Current neuron $>=$ winning neuron) then it is suspicious region other wise neglect it

\section{IMPLEMENTATION}

A self-organizing map consists of components called nodes or neurons. Associated with each node is a weight vector of the same dimension as the input data vectors and a position in the map space. The usual arrangement of nodes is a regular spacing in a hexagonal or rectangular grid. The self-organizing map describes a mapping from a higher dimensional input space to a lower dimensional map space. The procedure for placing a vector from data space onto the map is to find the node with the closest weight vector to the vector taken from data space and to assign the map coordinates of this node to our vector.

Euclidean distance to all weight vectors is computed. The neuron with weight vector most similar to the input is called the best matching unit (BMU). The weights of the BMU and neurons close to it in the SOM lattice are adjusted towards the input vector. The magnitude of the change decreases with time and with distance from the BMU. The update formula for a neuron with weight vector $\mathbf{W v}(\mathrm{i})$ is

$$
\begin{array}{r}
\mathbf{W v}(\mathrm{i}+1)=\mathrm{Wi}(\mathrm{i}+1)=\mathrm{wi}(\mathrm{i})+\mathrm{hci}(\mathrm{I}) *[(\mathrm{x}(\mathrm{i})- \\
\mathrm{w}(\mathrm{i}))]
\end{array}
$$

Here hci is neighborhood function to calculate it

$$
\mathrm{h}(\mathrm{i})=\mathrm{h}(\mathrm{rc}-\mathrm{r} 1) * \mathrm{a}(\mathrm{i}) * \text { alpha }
$$

$$
\text { Here } \mathrm{rc}-\mathrm{r} 1=\text { current neuron-next current neuron }
$$

$$
\mathrm{a}(\mathrm{i})=\operatorname{sigma} 0 * \exp (-\mathrm{i} / \mathrm{nsm})
$$

Initialize the variable

Sigma $=$ number of neighborhood pixels ( 8 or 24 or 48 or 80 or 120 )

$$
\begin{aligned}
& \text { if sliding window size }(3 * 3=8),(5 * 5=24),(7 * 7=48),) \\
& (9 * 9=80),(11 * 11=120) \\
& \text { Sigma N= Sigma } 0 * \exp (-\mathrm{i} / \text { taul })
\end{aligned}
$$

Taul= total number of pixels $/ \log ($ neighborhood number of pixel)

Similarly find the sigma value for each and every pixel This process is repeated for each input vector for a (usually large) number of cycles $\boldsymbol{\lambda}$. The network winds up associating output nodes with groups or patterns in the input data set. If these patterns can be named, the names can be attached to the associated nodes in the trained net. 
The winning neuron formula is

$$
\|\mathrm{x}-\mathrm{wc}\|=\operatorname{maxi}\{[=\mathrm{x}-\mathrm{wi}]\}
$$

$\mathrm{X}$ is a neuron,wi is the weight vector

\section{RESUlt AND ANALYSIS}

Table1 shows the result of image segmentation of HSOM .In any computer aided analysis ,the execution time is one of the important parameters of medical image segmentation .In these result, we have calculated the number of tumor cells of different neighborhood pixel of $3 \times 3,5 \times 5$, $7 \times 7,9 \times 9,11 \times 11$ windows. In that $3 \times 3$ window is chosen based on the high contrast than $5 \times 5,7 \times 7,9 \times 9$, and $11 \times 11$. TABLE 1 WinNING NEURON, NuMBER OF SEgMENTED PIXEL, EXECUTION TIME, WEIGHT

\begin{tabular}{|l|c|c|c|c|}
\hline & $\begin{array}{c}\text { Winning } \\
\text { neuron }\end{array}$ & $\begin{array}{c}\text { Number } \\
\text { of seg } \\
\text { pixel }\end{array}$ & $\begin{array}{c}\text { Exe } \\
\text { time }\end{array}$ & weight \\
\hline $3 \times 3$ & 209 & 795 & 13.76 & 14 \\
\hline $5 \times 5$ & 201 & 1073 & 14.96 & 8 \\
\hline $7 \times 7$ & 194 & 1285 & 15.20 & 15 \\
\hline $9 \times 9$ & 186 & 1594 & 11.05 & 23 \\
\hline $11 \times 11$ & 177 & 1881 & 11.53 & 32 \\
\hline
\end{tabular}

Figure 3 shows the tested segmented image with various neighborhood pixels the original image of(256x256).

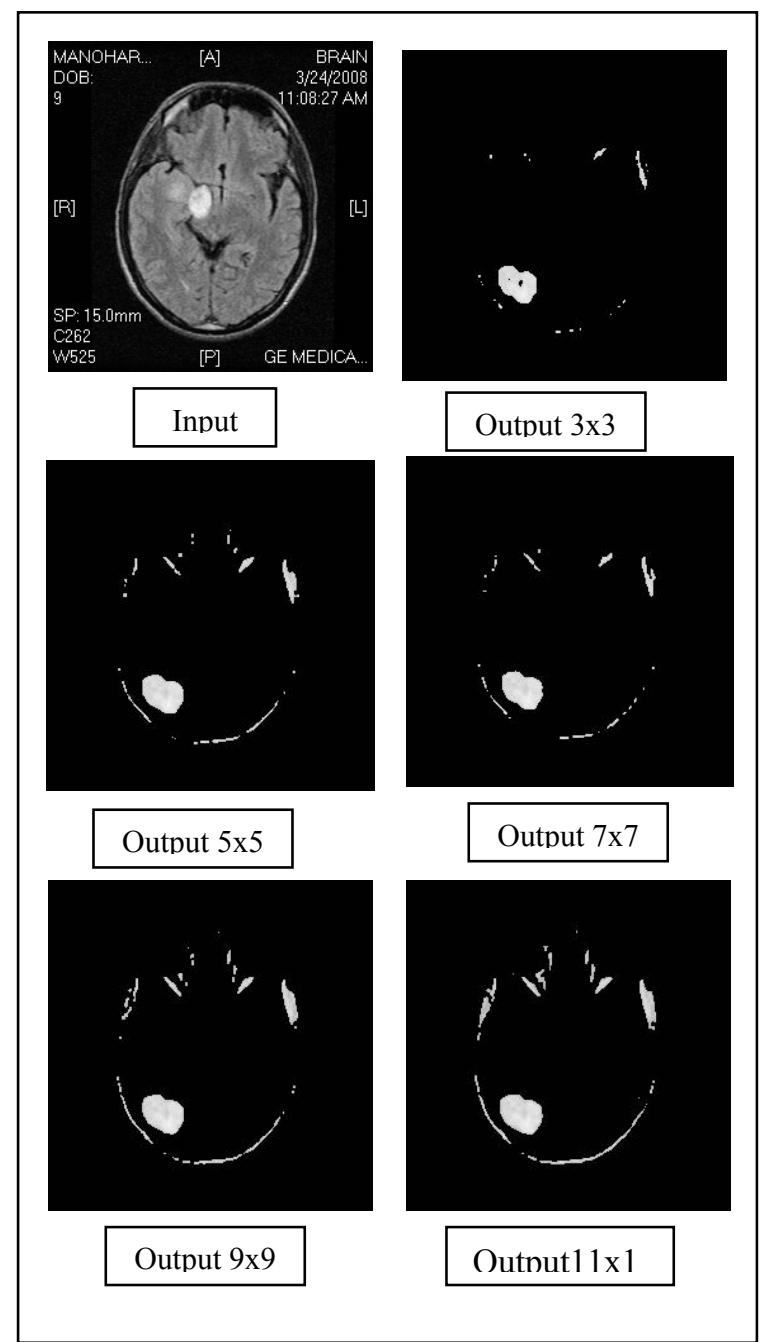

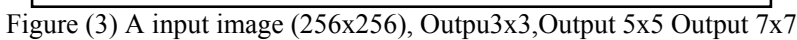
Output $9 \times 9$ Output $11 \times 11$

\section{PERFormanCE ANALYSIS}

It is very difficult to measure the performance of enhancement objectively. If the enhanced image can make observer perceive the region of interest better, then we can say that the original image has been improved. Here we are giving input image in that neighborhood pixel of $3 \times 3,5 \times 5$, $7 \times 7,9 \times 9,11 \times 11$ windows are analyzed. In that $3 \times 3$ window is chosen based on the high contrast than $5 \times 5,7 \times 7,9 \times 9$, and $11 \times 11$. Figure $(4 \mathrm{c})$ shows the weight vector for HSOM is $3 \times 3$ is $14,5 \times 5$ is $8,7 \times 7$ is $15,9 \times 9$ is 23 and $11 \times 11$ is 32 .Figure (4a) shows the Execution time in HSOM of $3 \times 3$ is $13.76,5 \times 5$ is $14.96,7 \times 7$ is $15.20,9 \times 9$ is 11.05 and $11 \times 11$ is 11.53 . Figure (4b) shows the number of segmented pixel in HSOM of $3 \times 3$ is $795,5 \times 5$ is $1073,7 \times 7$ is $1285,9 \times 9$ is 1594 and $11 \times 11$ is 1881 . (4c) shows the wining neuron for HSOM is $3 \times 3$ is 209 , $5 \times 5$ is $201,7 \times 7$ is $194,9 \times 9$ is 186 and $11 \times 11$ is 177 .

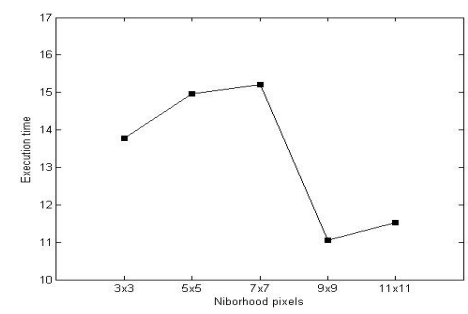

Fig(4a) relationship between execution time and neighborhood pixels

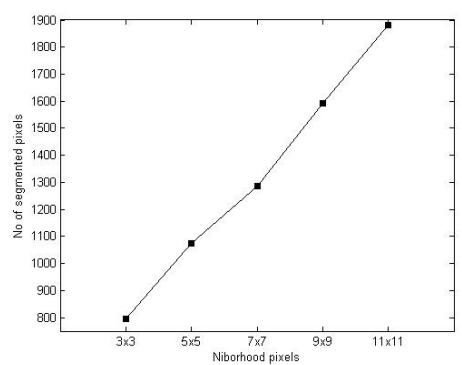

Fig(4b) relationship between no of segmented pixel and neighborhood pixels The weight of neuron

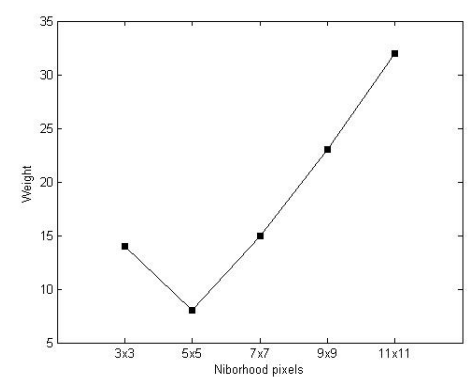

Fig(4c) relationship between weight and neighborhood pixels

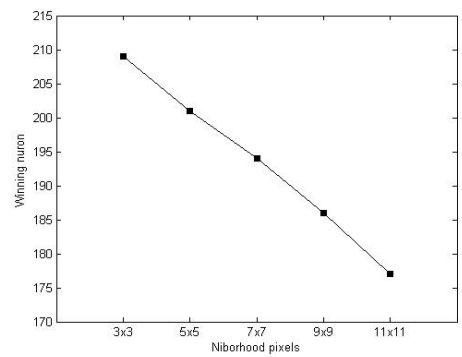

Fig(4d) relationship winning neuron and neighborhood pixels The above $3 \times 3,5 \times 5,7 \times 7,9 \times 9,11 \times 11$ windows are 
analyzed .In that $3 \times 3$ window is chosen based on the high contrast than $5 \times 5,7 \times 7,9 \times 9$, and $11 \times 11$.

\section{CONCLUSION}

Relevance of these techniques is the direct clinical application for segmentation. We studied the performance of the MRI image in terms of weight vector, execution time and tumor pixels detected. We have described several methods in medical image processing and discussed requirements and properties of techniques in brain tumor detection .This paper is used to give more information about brain tumor detection and segmentation. The target area is segmented and the evaluation of this tool from the doctor, whom the project is cooperated with, is positive and this tool helps the doctors in diagnosis, the treatment plan making and state of the tumor monitoring. In future ,the system should be improved by adapting more segmentation algorithm to suit the different medical image segmentation.

\section{REFERENCES}

[1] Ahalt, S.C., A.K. Krishnamurthy, P. Chen and D.E.Melton, 1990. "Competitive learning algorithms for Vector quantization," Neural Networks 3 (3): 277-290.

[2] Alirezaie, J., M.E. Jernigan and C. Nahmias, 1997."Neural Network based segmentation of MagneticResonance Images of the Brain", IEEE Trans. Nuclear Science, 44 (2): 194-198.

[3] Aidyanathan M, Clarke L.P, Velthuizen R.P, Phuphanich S, Bensaid A.M, Hall L.O, Bezdek J.C, Greenberg H, Trotti A, Silbiger M,:"Comparison of Supervised MRI Segmentation methods for Tumor Volume Determination During Therapy", Pergamon, Magnetic Resonance Imaging,vol.13,no.5,pp,719728,1995 .

[4] Bilbro, G., M. White and W. Snyder,. "Image segmentation with neurocomputers", In: R. Eckmiller and C.van der Malsburg (eds.), Neural Computers, NATO AS1 Series, (Springer-Verlag, Berlin, Germany), $41: 7$ 1-79,1987.

[5] Chunyan Jiang,Xinhua Zhang,Wanjun Huang,Christoph Meinel.:"Segmentation and Quantification of Brain Tumor,"IEEE International conference on Virtual Environment,Human-Computer interfaces and Measurement Systems, USA, 12-14, July 2000.

[6] DeSieno, D., 1988. "Adding a conscience to competitive learning", Proceeding of IEEE the Second International Conference on Neural networks(ICNN88) 1: 117- 124.[20]

[7] Fu, K.S. and J.K. Mui, 1981. "A survey on image segmentation", Pattern ecognition, 13: 3-16.

[8] Gonzalez, R.C. and R.E. Woods, 2002. "Digital image processing", Pearson Education, 2002.

[9] G. Papadourakis, G. Spanoudakis, A. Gotsias, "Application of neural networkssin short-term stock Price forecasting", in: Proceedings of the 1 International Workshop Neural Networks in the Capital Markets, 1993.

[10] G. Bandyopadhyay, S. Chattopadhyay, "Single hidden layer Artificial Neural Network models versus multiple linear regression model in forecasting the time series of total ozone", International Journal of Environmental Science and Technology 4 (1) (2007) 141-149.

[11] G. Chattopadhyay “Feed forward artificial neural network model to predict the average summerGeophysica 55(3): 369-38,.2007.
[12] G. Chattopadhyay and S. Chattopadhyay, "Autoregressive forecast of monthly total ozone concentration"A neurocomputing approach, Computers and Geosciences, 35, 1925-1932,2009.

[13] Gendy, G. Kothapalli, A. Bouzerdoum, "A fast algorithm for image restoration using a recurrent neural network with boundconstrained quadratic optimization" in: Proceedings of the 7th Australian and New Zealand Intelligent Systems Conference, 2001, pp. 111-115.

[14] Haralick, R.M. and L.G. Shapiro, "Survey ,image segmentation techniques", Computer Vision, Graphics Image Process, 29: 100132,1985 .

[15] Hillips,W.E, Velthuizen R.P, Phuphanich S, L.O, Clarke L.P, Silbiger ,:"Application of fuzzy C-Means Segmentation Technique for tissue Differentlation in MR Images of a hemorrhagic Glioblastoma Multiforme “, Pergamon,Megnetic Resonance Imaging, Vol.13, 1995.

[16] Karnan.M,Logeswari.T,Selvanayaki.K,:"'Introduction to detection of Automatic brain tumor through MRI",Proceedings of National Conference and Wokshop on Soft and Intelligent Computing,2325,jan 2008.

[17] Kohonen, T., 1988. "Self-Organization and Associative Memory", 2nd Edition (Springer-Verlag, Berlin, Germany.

[18] Kwok, T., R. Smith, S. Lozano and D. Taniar, "Parallel fuzzy cmeans clustering for large data sets", In Burkhard Monien and Rainer Feldmann, editors, EUROPAR02, 2400: 365-314,2002.

[19] Lin, W., E. Tsao and C. Chen,. "Constraint satisfaction neural networks for image segmentation", In: T.Kohonen, K. Mkisara, 0. Simula and J. Kangas (eds.), Artificial Neural Networks (Elsevier Science Publishers), pp: 1087-1090,1.991

[20] Naylor, J. and K.P. Li,. "Analysis of a neural network algorithm for vector quantization of speech Parameters", Proceeding of the 1st Annual INNS Meeting, pp: 310-315,1988.

[21] Parra, C.A., K. Iftekharuddin and R. Kozma, ."Automated Brain Tumor segmentation and pattern recognition using ANN",Computational Intelligence Robotics and Autonomous Systems, 2003 .

[22] Pal, N.R. and S.K. Pal, "A review on image segmentation techniques," Pattern Recognition 26(9): 1277-1294,1993.

[23] sai .C, Manjunath B.S,Jagadeesan.R,:"Automated Segmentation of brain MR Images",Pergamon,Pattern Recognition, Vol 28, No 12, March 1995.

[24] Samet, H., . "The Design and Analysis of Spatial Data Structures" (Addison-Wesley Pub. Co., Reading, MA) 1990.

[25] Sahoo, P.K., S. Soltani, A.K.C. Wong and Y.C. Chen, 1988. "A survey of thresholding techniques", Computer Vision, Graphics Image Process. 41: 233-260.

[26] Scherf, A. and G. Roberts, "Segmentation using neural networks for automatic thresholding," in: S. Rogers (ed.), Proc. SPIE Conference on Applications of Artificial Neural Networks (Orlando, FL, 1294), pp: 118-124,1990.

[27] hanShen,WilliamSandham,MalcolmGranet,Annette Sterr.:'MRI Fuzzy Segmentation of Brain Tissue Using Neighbourhood Attraction with Neural-Network Optimization",IEEE Transcations on Information Technology in BiomedicineVol.9,No.3,Sep 2005.

[28] S. Murugavalli1, V. Rajamani," A high speed parallel fuzzy c-mean algorithm for brain tumor segmentation", BIME Journal, Volume (06), Issue (1), Dec., 2006.

[29] . Murugavalli1 , V. Rajamani,” An Improved Implementation of Brain Tumor Detection Using Segmentation Based on Neuro Fuzzy Technique" Journal of Computer Science 3 (11): 841-846, 2007.

[30] Suchendra M. Bhandarkar, Jean Koh b, Minsoo Suk b," Multiscale image segmentation using a hierarchical self-organizing map", Neurocomputing $14 \quad$ (1997) 241-272. 


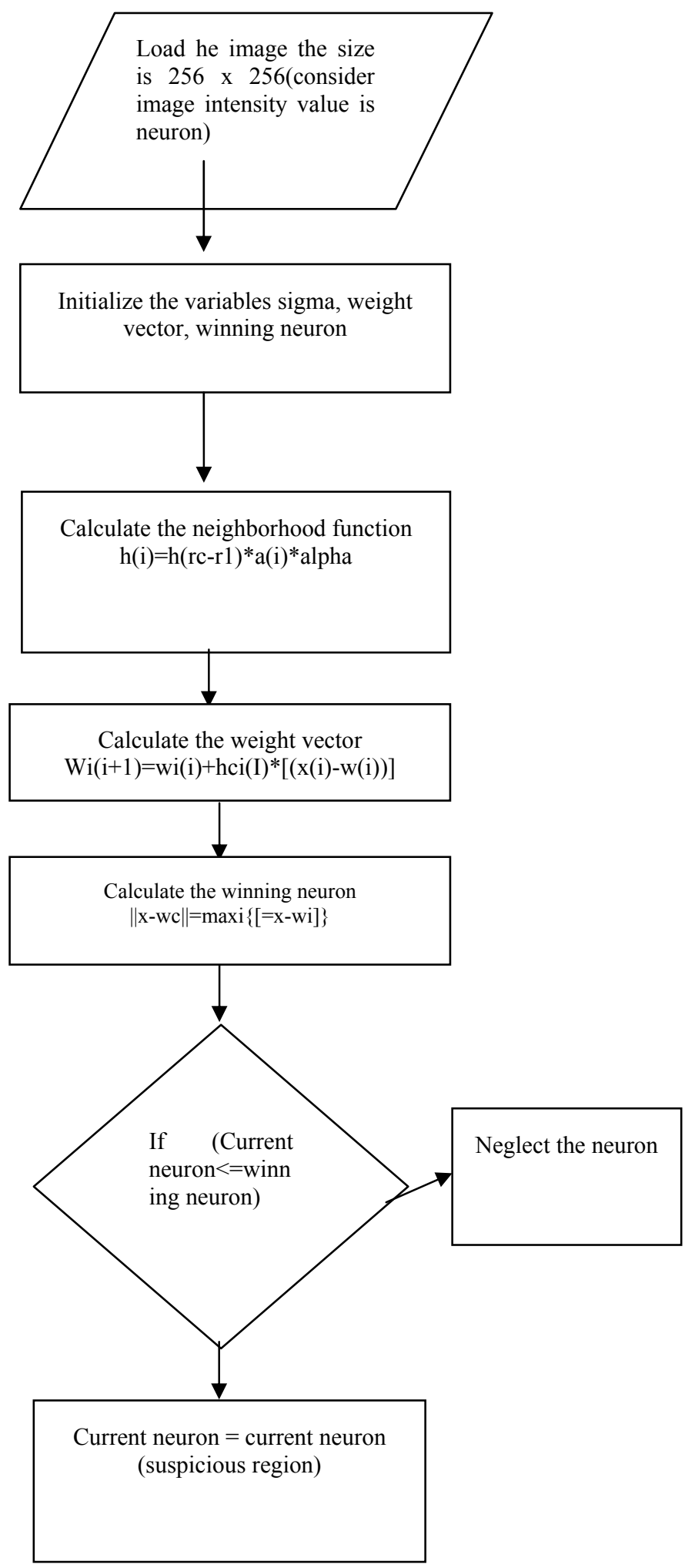

Fig (1) Flowdigram HSOM for detection of Brain Tumor 